\title{
Optimal Timing of VT Ablation for Patients with ICD Therapies
}

\author{
Andrea Radinovic $^{1}$ (D) $\cdot$ Francesca Baratto $^{1} \cdot$ Paolo Della Bella $^{1}$ \\ Published online: 9 July 2020 \\ (C) Springer Science+Business Media, LLC, part of Springer Nature 2020
}

\begin{abstract}
Purpose of Review Optimal timing for catheter ablation of ventricular tachycardia is an important yet unresolved subject. While it is clear that it is indicated with relatively advanced disease, it is still uncertain how early it should be recommended. In this review, we will focus on the status of timing of catheter ablation for VT in patients with ICD therapies.

Recent Findings The latest expert consensus statement added a new timing indication for catheter ablation after the first episode of monomorphic VT, in patients with ischemic heart disease and an ICD.

Summary Early referral for catheter ablation reduces the number of VT recurrences; however, an impact on mortality has not been demonstrated yet. Guidelines and real-world data alike show an increasing trend to refer patients after the first VT episode in ICD patients. Randomized clinical trials powered to assess mortality are essential in order confirm the beneficial effects of an early strategy.
\end{abstract}

Keywords Catheter ablation $\cdot$ Ventricular tachycardia $\cdot$ Ablation timing $\cdot$ Implantable cardioverter-defibrillator $\cdot$ Antiarrhythmic drugs

\section{Introduction}

Ventricular tachycardia (VT) is an important cause of morbidity and mortality in patients with structural heart disease. Implantable cardioverter-defibrillators (ICDs) enhance survival by effectively terminating VTs; however, ICD shocks are associated with an increase in mortality and a reduction of quality of life [1-5]. One of the main approaches to reduce the risk of shocks is the optimization of ICDs by programming high-rate and delayed therapy windows [6, 7]; nevertheless, shocks are not eliminated, and additional therapies are often required to prevent such events. The main two options are antiarrhythmic drug (AAD) therapy and catheter ablation

This article is part of the Topical Collection on Invasive Electrophysiology and Pacing

Andrea Radinovic

radinovic.andrea@hsr.it

Francesca Baratto

baratto.francesca@hsr.it

Paolo Della Bella

dellabella.paolo@hsr.it

1 Department of Cardiac Electrophysiology and Arrhythmology, IRCCS Ospedale San Raffaele, Via Olgettina 60, Milan, Italy
(CA). Different antiarrhythmic drugs have been associated with increased mortality in patients with structural heart disease; some can be ineffective at controlling ventricular arrhythmias or can be poorly tolerated [8-10]. The only drugs that have shown a reduction in VT episodes, in randomized clinical trials (RCTs), are amiodarone and sotalol. RCTs have demonstrated that these drugs can reduce VT recurrences in patients with an ICD; however, their efficacy is moderate, with no benefit on overall mortality and significant side effects [11-15]. Nonetheless, based on the current expert consensus statement $[16 \bullet \bullet]$, referral for catheter ablation is usually prompted by failure of AADs to control VT episodes. In this review, we will focus on the status of timing of catheter ablation for VT in patients with ICD therapies.

\section{Guidelines}

The latest expert consensus [16••] Class I indications to catheter ablation for patients with ischemic heart disease include (1) ablation of VT storm refractory to AAD therapy; different papers demonstrated a benefit in terms of recurrences and mortality in this group [17-19]. A meta-analysis [20] on patients undergoing catheter ablation for VT storm showed that the odds of death were four times higher after a failed procedure compared with those with a successful one, and that 
failure of the acute procedure carried a high mortality risk. (2) In patients with recurrent VT despite chronic amiodarone therapy, catheter ablation is recommended in preference to escalating AAD therapy. In the VANISH randomized clinical trial [21], a significantly lower rate of the composite primary outcome of death, ventricular tachycardia storm, or appropriate ICD shock was observed among patients undergoing catheter ablation than among those receiving an escalation in antiarrhythmic drug therapy. (3) Patients with ischemic heart disease and recurrent VT, despite AAD therapy or when AAD therapy is contraindicated or not tolerated. This is based on prospective trials [22-24] that showed how VT recurrences were associated with increased mortality, hospitalization and worse quality of life.

Indications for non-ischemic cardiomyopathy (NICM) have strengthened compared to the 2017 guidelines [25]. Recommendations gained a Class I indication based on long-term data [26-28], for electrical storm refractory to AAD therapy or recurrent VT where medications are ineffective, contraindicated, or not tolerated in order to reduce VT recurrences and ICD shocks.

Optimal timing of catheter ablation is an important yet unresolved subject. Guidelines started raising the issue in 2009 [29] by searching what density or frequency of VT warrants ablation, whether patients should receive prophylactic VT ablation after ICD implantation before recurrence of VT and if ablation could eliminate the need for an ICD. While prospective studies have focused on the aspect of prophylactic ablation and comparison with ICD therapy, there are currently no published RCTs addressing the first question. The only data available so far come from retrospective trials [30, 31] comparing an early ablation versus a late referral strategy in patients with structural heart disease; evidence was provided that an early strategy improved VT-free survival. All these studies had an influence on the evolution of the Guidelines leading to a new timing indication. First the 2015 ESC Guidelines [32] and recently the Expert consensus [16••] recommended catheter ablation with a class IIa and class IIb indication respectively, after a first episode of sustained VT in ischemic patients with an ICD (regardless of the type of ICD therapy).

\section{Real-World Data}

The attitude toward the timing of ablation tends to reflect locally available expertise and logistics. Data from the 2015 survey of the European Heart Rhythm Society (EHRA) report that ablation was the treatment of choice in ischemic cardiomyopathy (ICM) patients after the first ICD shock due to VT in $13.3 \%$ of Centers [33]. In 2017, a new EHRA survey was conducted [34], showing that patients with ischemic cardiomyopathy and impaired left ventricular ejection fraction
(LVEF), presenting with their first episode of hemodynamically stable sustained monomorphic VT, despite beta-blocker therapy, were treated with VT ablation in $62.0 \%$ of the responding centers; amiodarone was typically used as firstline treatment in $19.7 \%$. In patients with non-ischemic etiology, the preferred initial strategy was catheter ablation in $37.1 \%$ of the centers and amiodarone in $41.4 \%$. All centers $(100 \%)$ indicated that the appropriate treatment for a patient with ischemic cardiomyopathy presenting multiple appropriate ICD shocks despite long treatment with amiodarone would be VT ablation; this was recommended in $89.8 \%$ of patients with non-ischemic cardiomyopathy. The reported data show that an increasing number of centers across Europe perform catheter ablation after the first ICD shock, following the release of the 2015 ESC guidelines, despite a weaker strength of recommendation. Unfortunately, real-world data based on such large surveys from American centers are not available.

\section{VT Ablation Timing}

\section{Retrospective Trials}

Three retrospective trials have been published on the timing of VT ablation in patients with ICD therapies. Frankel et al. [30•] reported a single-center experience on 98 consecutive patients with sustained VT and structural heart disease (both ICM and NICM), referred for the first catheter ablation procedure. Ninety-six percent of patients had an ICD implanted prior to ablation. They were divided into two groups: late referrals were defined as those with 2 or more episodes of VT, with the first and most recent episode separated by at least 1 month; all others were considered early referrals. Patients referred late for VT ablation were more likely to be on high dose amiodarone. Long-term outcomes showed that the early referral group had improved VT-free survival compared to the late group. In multivariate modeling, only early referral retained independent, statistically significant association with VT-free survival. Dinov et al. [31] investigated a similar population of 300 patients. The timing to ablation groups were different: early ablation strategy (group 1), consisting of patients ablated within 30 days after the first documented VT; delayed ablation strategy (group 2), patients ablated between 1 month and 1 year after the initial VT; very late ablation strategy (group 3), CA was performed $>1$ year after the first documented VT. Patients referred for VT ablation within 30 days after the first documented VT were the only group who had improved both acute and long-term success rates. The authors also analyzed the impact of timing on cardiac mortality reporting that early ablation strategy was not associated with improved survival; however, VT recurrence was associated with cardiovascular mortality. Romero et al. [35] included 669 consecutive patients who presented for their first percutaneous catheter 
ablation of sustained monomorphic VT, in addition to ICM and NICM they also included subjects without structural heart disease. Their definition of early vs late referral was not defined by time but by the number of failed orally administered AADs before referral for catheter ablation; hence, timing between VT onset and CA were not available. Patients were divided into 2 groups: (1) failure of no AAD or a single AAD to control VT (single-drug failure) or (2) failure of $>1$ AAD to control VT (multidrug failure). They analyzed VTfree survival, cardiac transplantation free survival, and overall survival. The authors report that patients with NICM-related VT who were in the multidrug group had substantially worse outcomes of arrhythmia recurrence, heart transplantation, and mortality compared with patients referred for ablation following no drug failure or single-drug failure. Whereas, there was no influence in patients without structural heart disease. In the ICM group, multidrug failure was associated with worse ventricular arrhythmia free survival and overall survival; however, the association was weaker; this was predominantly influenced by worse outcomes in patients with failed amiodarone treatment and it is consistent with the VANISH study where any amiodarone failure was associated with worse outcomes in ICM.

A systematic review and meta-analysis of clinical outcomes of these three studies [36•] confirmed that early ablation was associated with an improvement in VT-free survival in patients with both ICM and NICM, as well as fewer complications. However, there was no significant association with lower mortality, probably because they were underpowered to detect a mortality benefit due to limited follow-up, the limited sample size, and small number of deaths. One of the critical aspects underlined in this analysis is that there is no standard definition of early or late referral. Factors like clustering, frequency, and severity of VT are not taken into account, making the comparison less stringent.

However, retrospective data provide a solid background to support the need for prospective randomized studies to confirm the benefits of an early referral for catheter ablation of ventricular tachycardia in structural heart disease.

\section{Prospective Trials}

Randomized controlled trials addressed the issue of prophylactic catheter ablation in patients with ischemic cardiomyopathy. Even though the majority of patients included did not have an ICD implanted at enrollment, these are important data on early referral of catheter ablation after a VT episode and the impact on subsequent ICD therapy. The SMASH-VT [37] study included 128 patients with a history of myocardial infarction, who underwent ICD implantation for secondary prevention (for ventricular fibrillation, hemodynamically unstable ventricular tachycardia, syncope with inducible ventricular tachycardia during invasive electrophysiological testing), or received an appropriate therapy after primary prevention indications. Patients were randomized to ICD implantation alone or ICD implantation plus catheter ablation; during follow-up, no patient received an antiarrhythmic agent (other than betablockers). Patients in the ablation group had a $73 \%$ reduction in the risk of receiving subsequent ICD shocks; there was even a trend toward decreased mortality in the ablation group, although it was not statistically significant. The VTACH trial [38] enrolled 107 patients with previous myocardial infarction who had an indication for an ICD as secondary prevention after documented stable clinical VT. Patients were randomized to either VT ablation followed by ICD implantation or implantation alone. Drug management during follow-up was at the discretion of the investigator. Patients in the ablation group had a longer time free of any VT or VF recurrence, fewer appropriate ICD shocks, and fewer hospital admissions for cardiac reasons than did patients with an ICD only; there was no difference in mortality between the groups. The SMS study [39] published by the same group was a complement to the VTACH trial, as it had a similar design but it enrolled 111 patients with unstable ventricular arrhythmias. In contrast with the two previous studies, prophylactic catheter ablation did not prolong the time to first ventricular arrhythmia recurrence. The reasons for this difference were probably multiple, including different ICD programming, mapping and ablation strategy, drug management fluctuations, and the asymmetrical loss to follow-up of the two arms. However, catheter ablation was associated with a $>50 \%$ reduction in the total number of ICD therapies throughout follow-up.

The CALYPSO pilot randomized study [40] aimed at testing whether early use of catheter ablation of VT is superior to AAD introduction at reducing mortality. Twenty-seven enrolled patients with IHD and an ICD were randomized to CA or AAD if they received an ICD intervention $(\geq 1$ ICD shock or $\geq 3$ ATPs). The limited sample size and the follow-up period ( 3 months) did not show any significant difference in the two arms. This was a useful pilot study that showed the difficulty to conduct trials for early referrals, because of substantial obstacles to enrolling patients in a multicenter RCTs comparing a strategy of early CA with a strategy of AAD. This was due to the common clinical practice of starting $\mathrm{AAD}$ before ablation, resulting in a population referred for CA with progressed disease when drugs have already failed. An important alternative design was in the VANISH study [21] where patients were randomized to CA or escalated drug therapy after failing an AAD, showing a significant benefit in the composite endpoint of death, VT storm, or ICD shock in the ablation arm in relatively advanced disease patients, failing however to show improvement with respect to mortality alone. This led to the ongoing VANISH 2 trial (ClinicalTrials. gov Identifier: NCT02830360) that aims to define the best first-line therapy for VT, randomizing patients with a prior myocardial infarction to catheter ablation or AAD 
introduction after a VT event. The objective is to evaluate a reduction in the composite outcome of death, appropriate ICD shocks, VT storm, or treated sustained VT below the detection rate of the ICD.

A meta-analysis that included these five published RCTs [41] showed that patients in the ablation arm were significantly less likely to experience ICD shocks, VT storm, and cardiac hospitalization. However, no benefit was seen in all-cause mortality.

The latest RCT published on this topic is the BERLIN VT [42•]; the main difference with the previously randomized trials is that it compares prophylactic catheter ablation to a deferred strategy, instead of non-ablative treatments. At enrollment, 159 patients were randomly assigned, to receive catheter ablation for VT either before ICD implantation or after the third appropriate ICD shock for VT. The primary composite endpoint was all-cause death and hospitalization for heart failure or arrhythmia, and secondary end points were recurrent episodes of VT and change in quality of life at 1 year. The study was interrupted for futility because at the interim analysis, the primary endpoint increased sufficiently in the prophylactic treatment arm reaching the futility stopping boundary. The authors concluded that preventive VT ablation before ICD implantation did not reduce mortality or hospitalization for arrhythmia or worsening heart failure during a 1year of follow-up, compared with the deferred ablation strategy. The lack of benefit of prophylactic catheter ablation in this study is not surprising as it seems. A combination of factors including the study design and population made the trial underpowered to examine a hard endpoint like mortality. The population had a relatively high LVEF ( $\geq 30 \%$ ), ICDs were programmed to reduce shocks, the use of AADs was not pre-specified, and the follow-up period was too short to discern between such groups. Chance seemingly played a role as well; all-cause mortality was reported in 6 patients in the preventive ablation group including 1 case of endocarditis post TAVI procedure, 4 non-cardiac deaths, and one of unknown cause, whereas the only death cases reported due to arrhythmias were in the deferred ablation arm. An interesting aspect on the timing of ablation comes from the observation that, in the deferred ablation group, most physicians and patients did not adhere to the protocol and catheter ablation was performed after 1 or 2 ICD shocks. This echoes a shared opinion by some electrophysiologists in the community that three shocks may be too long to wait and reflects the realworld records available.

\section{What We Learned from RCTs: In Medio Stat Virtus}

Evidence from recent RCTs points to a lack of net benefit of prophylactic ablation of VT at the time of ICD implantation. Even though there is a reduction in the number of recurrences, there is no advantage in terms of mortality, and it does not eliminate the need for an ICD no matter how early it is performed. In view of its unfavorable cost-benefit ratio, it is unlikely that prophylactic catheter ablation of VT will be applied in clinical practice. On the other hand, we learned that deferring the timing of ablation to an undefined future definitely poses an increased risk for arrhythmia recurrence, with a probable adverse effect on the subsequent clinical course. Hence, what future randomized clinical trials need to address is when to perform ablation between these two extreme timings, even more so because that is where the majority of patients are. Patient recruitment of this population in RCTs is challenging. First, the difficulty to enroll subjects at the right time, since referral to large academic centers occurs when AADs have failed and the disease is more advanced. Second, limited duration of the available follow-up time, comorbidities and advanced age may concur to overall non-cardiac mortality, thus increasing the difficulty of proving a mortality benefit. Composite endpoints including mortality can certainly increase the number of events and shorten the follow-up period but they need to be chosen carefully.

In our opinion, the right timing for catheter ablation would be after ICD implantation but before AAD therapy, and an ICD shock could be the pivotal point in the natural history of the disease for randomization and risk stratification, being an intervention with prognostic value.

\section{Prophylactic Versus True Timing Ablation Trials}

The RCTs published so far were a comparison of strategies; however, none were true timing trials designed to randomize patients with ICDs in two different moments of the natural history of VT.

The issue of timing of ablation in patients with VT recurrences leading to an ICD shock is investigated in the ongoing PARTITA randomized clinical trial (Does Timing of VT Ablation Affect Prognosis in Patients With an Implantable Cardioverter-defibrillator? ClinicalTrials.gov Identifier: NCT01547208). This is the only study to randomize patients after the first ICD shock reflecting real-world practice patterns. It is a prospective randomized multicenter study, where patients are followed-up with automatic daily remote monitoring. All patients with an ICD, with ischemic and non-ischemic cardiomyopathy (both primary and secondary prevention), are enrolled and followed-up until their first appropriate ICD shock (Phase A). After the first appropriate shock delivered for VT, patients are randomized (Phase B) to either the control group (ablation after the next electrical storm) or the immediate VT ablation arm. The primary endpoint is a composite of heart failure hospitalization and death from any cause; the follow-up period is 2 years after the ICD shock. The secondary endpoints include the correlation of the first appropriate ICD shock to the burden of previous VTs (Phase A), cardiac death, recurrent electrical storm, and recurrent VT (Phase B). 
The study began in September 2012 and at the time of writing, there are 16 European sites actively enrolling. Non-ablative policies of the protocol aim to give insight on the natural history of ventricular tachycardia after ICD implant. Abstention from the use of AADs will help observe the natural progression and burden of the arrhythmia with the use of remote monitoring. Standardized device programming, reflecting current clinical practice, with long detection intervals favoring antitachycardia pacing (ATP), will help analyze the predictive role of ATPs on ICD shocks.

Similarly to other trials, the primary mortality endpoint is composite and in this case includes heart failure hospitalization; the fact that death from any cause is used instead of cardiac death could make it more challenging to demonstrate a net benefit. Secondary endpoints however target the specific relationship between timing of catheter ablation and cardiac death, which is the missing link that is needed.

\section{Clinical Decision-making}

The current practice in our center is shown in Fig. 1. In patients naïve to VT (Fig. 1(A)), we tend not to use AADs unless they are required for other arrhythmias, up-titrate betablockers when ICD interventions occur, and recommend the use of home monitoring. In case of a shock, we reprogram the device when possible to delay therapies and perform catheter ablation when $>2$ shocks occur in 12 months. Therapeutic strategies after failed VT ablation are less standardized and depend on the clinical characteristics of the patient and of the ventricular tachycardia. Multicenter data have shown that a repeated catheter ablation is usually performed after multiple electrical shocks or electrical storm and having failed amiodarone and multiple other AADs, more commonly in NICM [43]. In our center (Fig. 1(B)), we usually discharge patients after $\mathrm{CA}$ with a home monitoring device, an optimized medical therapy without AADs. In case of ATPs, beta-blockers are up-titrated when possible. In case of an ICD shock, we tend to perform CA earlier (after 1 shock) in patients with an ischemic substrate, whereas we prefer AAD therapy in NICM patients and save catheter ablation after further recurrences.

In case of refractory VT storm to all the above-mentioned treatments, surgical autonomic modulation is a reasonable option. The principle of sympathetic modulation is based on the fact that the autonomic nervous system plays an important role in triggering and maintaining ventricular tachycardia. Indeed, beta-adrenergic receptor blockers are a standard therapy used for neuromodulation that aim to block sympathetic transmission. Surgical interventions such as cardiac sympathetic denervation (CSD) that target the stellate ganglia by videoassisted thoracoscopy can reduce the burden of sustained VT and ICD shocks [44]. The 2017 Guidelines [25] and the Expert consensus $[16 \bullet \cdot$ consider this emerging therapeutic option reasonable in patients with VT storm after betablockers, AADs, and VT ablation have failed.

\section{Management of VT Patients in the COVID-19 Era}

In this historic moment, it is timely to analyze how the management of patients with VT is going to change due to the Coronavirus pandemic.

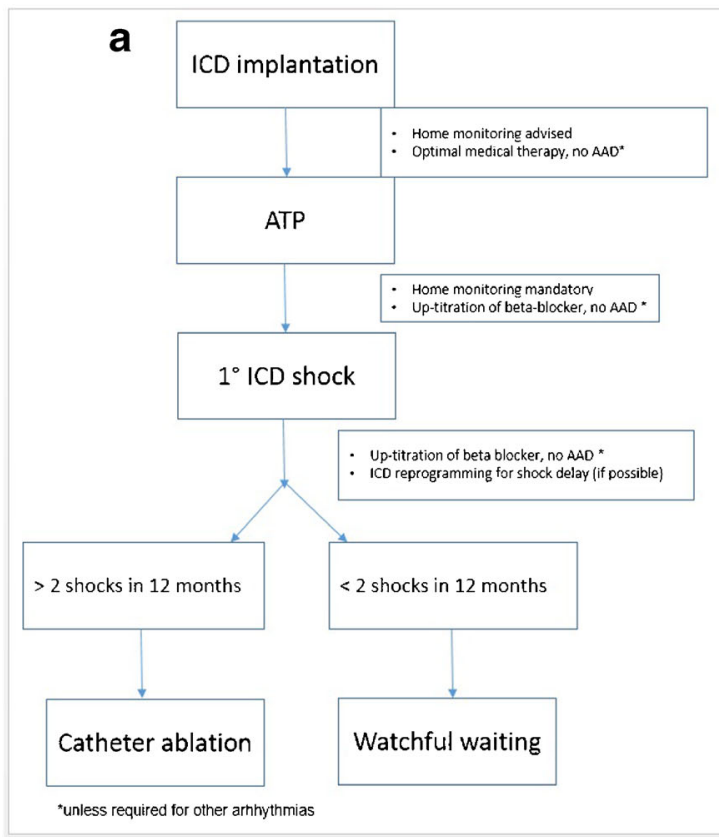

Fig. 1 Flowchart demonstrating current clinical practice in our center for the timing of VT ablation in VT-naive patients (A) and after catheter ablation recurrence (B). AAD, antiarrhythmic drug; ATP, antitachycardia

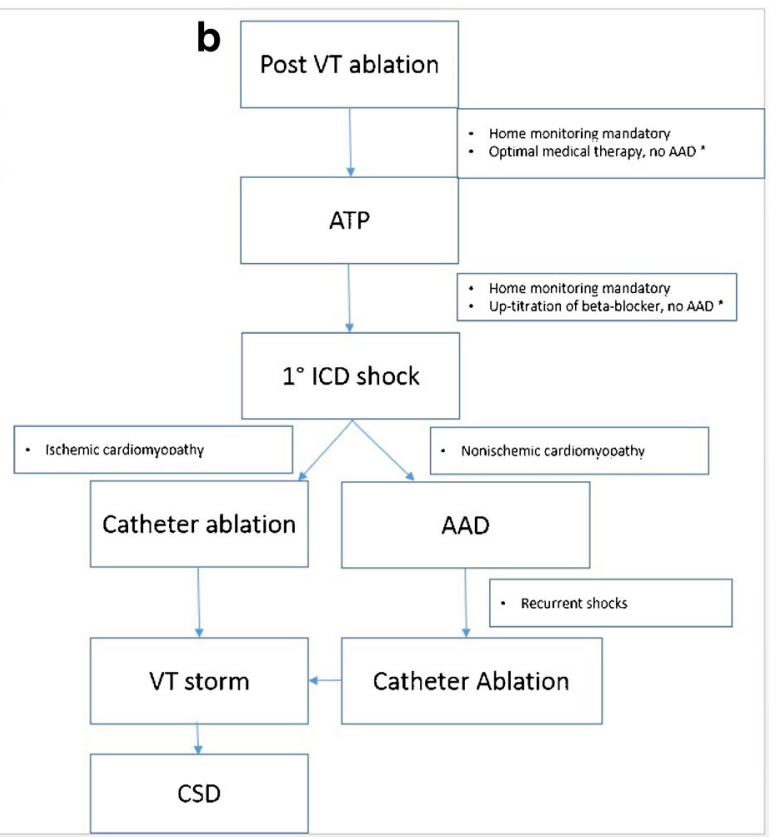

pacing; CSD, cardiac sympathetic denervation; ICD, implantable cardioverter-defibrillator; VT, ventricular tachycardia 
Following the outbreak, the common decisional pattern of all European Centers was to adapt to the National Health Authorities request to shut down elective procedures, thereby allowing a shift of personnel and equipment resources in favor of the Intensive and Nonintensive Covid Care Units. As a result, and according to a recently published survey from our Institution [45], the number of non-urgent procedures dropped almost to zero. The number of VT ablation procedures, thereby performed only on an emergency indication, decreased roughly by $85 \%$ with respect to the pre-outbreak metrics.

\section{What Will the Foreseeable Future Look like?}

Patient visits and especially referrals will become more difficult; this will also have an impact on enrolment and follow-up in trials. Due to the important impact on morbidity and mortality of ventricular tachycardia, it will be our duty to intensify the activity of remote monitoring, to ensure prompt recognition of evolving arrhythmia patterns, chasing "warming" patterns, setting "earlier" indications to VT ablation as compared to the current behavior, to avoid progressive deterioration of the arrhythmia and overall clinical course. Home monitoring will become crucial in evaluating the disease progression, adjusting therapy accordingly and shortening referrals.

Considering the interactions of Class III agents with drugs used for the treatment of viral infection, their use might be reduced due to their impact on QT prolongation. The resulting reduced shelter could lead to an increased susceptibility to arrhythmias that should be treated by catheter ablation.

Lastly, there is increasing evidence of elevated troponin levels in COVID-19 patients [46, 47]; while at the moment we are uncertain whether this could lead to a significant cardiac damage and substrate for ventricular tachycardia, we should be prepared to a potential increase in vulnerability to ventricular arrhythmias and risk stratification in this new scenario.

\section{Conclusions}

Indication to catheter ablation is usually recommended when AADs have failed to control VT recurrences and ICD shocks, in patients with a more advanced disease. There is a growing amount of evidence that an early referral for ablation reduces VT recurrences, though a benefit in terms of mortality has not been demonstrated yet. This however led to a Guideline indication to consider ICD patients for ablation after the first VT episode. Real-world data suggest that it is an increasingly common clinical practice to evaluate patients for catheter ablation earlier in the natural history, in order to avoid the demonstrated negative impact on patients' quality of life and possible side effects of drugs. Randomized clinical trials powered to assess mortality are essential in order confirm the beneficial effects of an early strategy.

\section{Compliance with Ethical Standards}

Conflict of Interest Andrea Radinovic and Francesca Baratto declare that they have no conflict of interest.

Paolo Della Bella reports grants and personal fees from Abbott and Biosense Webster, and grants from Biotronik and Boston Scientific.

Human and Animal Rights and Informed Consent This article does not contain any studies with human or animal subjects performed by any of the authors.

\section{References}

Papers of particular interest, published recently, have been highlighted as:

- Of importance

•. Of major importance

1. Moss AJ, Greenberg H, Case RB, Zareba W, Hall WJ, Brown MW, et al. Long-term clinical course of patients after termination of ventricular tachyarrhythmia by an implanted defibrillator. Circulation. 2004;110:3760-5. https://doi.org/10.1161/01.CIR.0000150390. 04704.B7.

2. Poole JE, Johnson GW, Hellkamp AS, Anderson J, Callans DJ, Raitt MH, et al. Prognostic importance of defibrillator shocks in patients with heart failure. N Engl J Med. 2008;359:1009-17. https://doi.org/10.1056/NEJMoa071098.

3. Schron EB, Exner DV, Yao Q, Jenkins LS, Steinberg JS, Cook JR, et al. Quality of life in the antiarrhythmics versus implantable defibrillators trial: impact of therapy and influence of adverse symptoms and defibrillator shocks. Circulation. 2002;105:589-94. https://doi.org/10.1161/hc0502.103330.

4. Noyes K, Corona E, Veazie P, Dick AW, Zhao H, Moss AJ. Examination of the effect of implantable cardioverterdefibrillators on health-related quality of life: based on results from the multicenter automatic defibrillator trial-II. Am J Cardiovasc Drugs. 2009;9:393-400. https://doi.org/10.2165/11317980000000000-00000.

5. Dunbar SB, Dougherty CM, Sears SF, Carroll DL, Goldstein NE, Mark DB, et al. Educational and psychological interventions to improve outcomes for recipients of implantable cardioverter defibrillators and their families: a scientific statement from the American Heart Association. Circulation. 2012;126:2146-72. https://doi.org/10.1161/CIR.0b013e31825d59fd.

6. Moss AJ, Schuger C, Beck CA, Brown MW, Cannom DS, Daubert $\mathrm{JP}$, et al. Reduction in inappropriate therapy and mortality through ICD programming. N Engl J Med. 2012 Dec 13;367:2275-83. https://doi.org/10.1056/NEJMoa1211107.

7. Gasparini M, Proclemer A, Klersy C, Kloppe A, Lunati M, Ferrer $\mathrm{JB}$, et al. Effect of long-detection interval vs standard-detection interval for implantable cardioverter-defibrillators on antitachycardia pacing and shock delivery: the ADVANCE III randomized clinical trial. JAMA. 2013;309:1903-11. https://doi.org/ 10.1001/jama.2013.4598.

8. IMPACT Research Group. International mexiletine and placebo antiarrhythmic coronary trial: I. Report on arrhythmia and other findings. J Am Coll Cardiol. 1984;4:1148-63. https://doi.org/10. 1016/s0735-1097(84)80133-3. 
9. Echt DS, Liebson PR, Mitchell LB, Peters RW, Obias-Manno D, Barker AH, et al. Mortality and morbidity in patients receiving encainide, flecainide, or placebo. The Cardiac Arrhythmia Suppression Trial. N Engl J Med. 1991;324:781-8. https://doi. org/10.1056/NEJM199103213241201.

10. Effect of the antiarrhythmic agent moricizine on survival after myocardial infarction. The cardiac arrhythmia suppression trial II investigators. N Engl J Med. 1992;327:227-33. https://doi.org/10.1056/ NEJM199207233270403.

11. Connolly SJ, Dorian P, Roberts RS, Gent M, Bailin S, Fain ES, et al. Comparison of $\beta$-Blockers, Amiodarone Plus $\beta$-Blockers, or Sotalol for prevention of shocks from implantable cardioverter defibrillators: the OPTIC Study: A Randomized Trial. JAMA. 2006;295:165-71.

12. Piccini JP, Berger JS, O'Connor CM. Amiodarone for the prevention of sudden cardiac death: a meta-analysis of randomized controlled trials. Eur Heart J. 2009 May;30:1245-53. https://doi.org/10. 1093/eurheartj/ehp100.

13. Bokhari F, Newman D, Greene M, Korley V, Mangat I, Dorian P. Long-term comparison of the implantable cardioverter defibrillator versus amiodarone: eleven-year follow-up of a subset of patients in the Canadian implantable defibrillator study (CIDS). Circulation. 2004;110:112-6. https://doi.org/10.1161/01.CIR.0000134957. $51747.6 \mathrm{E}$

14. Vorperian VR, Havighurst TC, Miller S, January CT. Adverse effects of low dose amiodarone: a meta-analysis. J Am Coll Cardiol. 1997;30:791-8. https://doi.org/10.1016/s0735-1097(97)00220-9.

15. Waldo AL, Camm AJ, de Ruyter H, Friedman PL, MacNeil DJ, Pauls JF, et al. Effect of d-sotalol on mortality in patients with left ventricular dysfunction after recent and remote myocardial infarction. The SWORD investigators. Survival With Oral d-Sotalol. Lancet. 1996;348:7-12.

16.• Cronin EM, Bogun FM, Maury P, Peichl P, Chen M, Namboodiri $\mathrm{N}$, et al. HRS/EHRA/APHRS/LAHRS expert consensus statement on catheter ablation of ventricular arrhythmias: executive summary. Heart Rhythm. 2019;17:e155-205. https://doi.org/10.1016/j.hrthm. 2019.03.014 This document provides the latest evidence based guidelines for catheter ablation of ventricular arrhythmias.

17. Carbucicchio C, Santamaria M, Trevisi N, Maccabelli G, Giraldi F, Fassini G, et al. Catheter ablation for the treatment of electrical storm in patients with implantable cardioverterdefibrillators: shortand long-term outcomes in a prospective single-center study. Circulation. 2008;117:462-9. https://doi.org/10.1161/ CIRCULATIONAHA.106.686534.

18. Deneke T, Shin DI, Lawo T, Bösche L, Balta O, Anders H, et al. Catheter ablation of electrical storm in a collaborative hospital network. Am J Cardiol. 2011;108:233-9. https://doi.org/10.1016/j. amjcard.2011.03.030.

19. Muser D, Liang JJ, Pathak RK, Magnani S, Castro SA, Hayashi T, et al. Long-term outcomes of catheter ablation of electrical storm in nonischemic dilated cardiomyopathy compared with ischemic cardiomyopathy. JACC Clin Electrophysiol. 2017;3:767-78. https:// doi.org/10.1016/j.jacep.2017.01.020.

20. Nayyar S, Ganesan AN, Brooks AG, Sullivan T, Roberts-Thomson $\mathrm{KC}$, Sanders P. Venturing into ventricular arrhythmia storm: a systematic review and meta-analysis. Eur Heart J. 2013;34:560-71. https://doi.org/10.1093/eurheartj/ehs453.

21. Sapp JL, Wells GA, Parkash R, Stevenson WG, Blier L, Sarrazin $\mathrm{JF}$, et al. Ventricular tachycardia ablation versus escalation of antiarrhythmic drugs. N Engl J Med. 2016;375:111-21. https://doi.org/ 10.1056/NEJMoa1513614.

22. Stevenson WG, Wilber DJ, Natale A, Jackman WM, Marchlinski FE, Talbert T, et al. Irrigated radiofrequency catheter ablation guided by electroanatomic mapping for recurrent ventricular tachycardia after myocardial infarction: the multicenter thermocool ventricular tachycardia ablation trial. Circulation. 2008;118:277382. https://doi.org/10.1161/CIRCULATIONAHA.108.788604.

23. Tanner H, Hindricks G, Volkmer M, Furniss S, Kühlkamp V, Lacroix D, et al. Catheter ablation of recurrent scar-related ventricular tachycardia using electroanatomical mapping and irrigated ablation technology: results of the prospective multicenter euro-VTstudy. J Cardiovasc Electrophysiol. 2010 Jan;21:47-53. https://doi. org/10.1111/j.1540-8167.2009.01563.

24. Marchlinski FE, Haffajee CI, Beshai JF, Dickfeld TL, Gonzalez $\mathrm{MD}, \mathrm{Hsia} \mathrm{HH}$, et al. Long-term success of irrigated radiofrequency catheter ablation of sustained ventricular tachycardia: post-approval THERMOCOOL VT trial. J Am Coll Cardiol. 2016 Feb 16;67: 674-83. https://doi.org/10.1016/j.jacc.2015.11.041.

25. Al-Khatib SM, Stevenson WG, Ackerman MJ, Bryant WJ, Callans DJ, Curtis AB, et al. 2017 AHA/ACC/HRS guideline for management of patients with ventricular arrhythmias and the prevention of sudden cardiac death: executive summary: a report of the American College of Cardiology/American Heart Association task force on Clinical Practice Guidelines and the Heart Rhythm Society. Heart Rhythm. 2018 Oct;15:e190-252. https://doi.org/10.1016/j.hrthm. 2017.10.035.

26. Tung R, Vaseghi M, Frankel DS, Vergara P, Di Biase L, Nagashima K, et al. Freedom from recurrent ventricular tachycardia after catheter ablation is associated with improved survival in patients with structural heart disease: an international VT ablation center collaborative group study. Heart Rhythm. 2015 Sep;12: 1997-2007. https://doi.org/10.1016/j.hrthm.2015.05.036.

27. Muser D, Liang JJ, Pathak RK, Magnani S, Castro SA, Hayashi T. Long-term outcomes of catheter ablation of electrical storm in nonischemic dilated cardiomyopathy compared with ischemic cardiomyopathy. JACC Clin Electrophysiol. 2017 Jul;3:767-78. https://doi.org/10.1016/j.jacep.2017.01.020.

28. Muser D, Santangeli P, Castro SA, Pathak RK, Liang JJ, Hayashi T. Long-term outcome after catheter ablation of ventricular tachycardia in patients with nonischemic dilated cardiomyopathy. Circ Arrhythm Electrophysiol. 2016;9:e004328.

29. Aliot EM, Stevenson WG, Almendral-Garrote JM, Bogun F, Calkins CH, Delacretaz E. EHRA/HRS Expert Consensus on Catheter Ablation of Ventricular Arrhythmias: developed in a partnership with the European Heart Rhythm Association (EHRA), a Registered Branch of the European Society of Cardiology (ESC), and the Heart Rhythm Society (HRS); in collaboration with the American College of Cardiology (ACC) and the American Heart Association (AHA). Heart Rhythm. 2009;6:886-933. https://doi. org/10.1016/j.hrthm.2009.04.030.

30. Frankel DS, Mountantonakis SE, Robinson MR, Zado ES, Callans DJ, Marchlinski FE. Ventricular tachycardia ablation remains treatment of last resort in structural heart disease: argument for earlier intervention. J Cardiovasc Electrophysiol. 2011;22:1123-8. https:// doi.org/10.1111/j.1540-8167.2011.02081 First study to compare an early vs late referral for catheter ablation.

31. Dinov B, Arya A, Bertagnolli L, Schirripa V, Schoene K, Sommer $P$, et al. Early referral for ablation of scar-related ventricular tachycardia is associated with improved acute and long-term outcomes: results from the heart Center of Leipzig ventricular tachycardia registry. Circ Arrhythm Electrophysiol. 2014;7:1144-51. https:// doi.org/10.1161/CIRCEP.114.001953.

32. Priori SG, Blomström-Lundqvist C, Mazzanti A, Blom N, Borggrefe M, Camm J, et al. 2015 ESC Guidelines for the management of patients with ventricular arrhythmias and the prevention of sudden cardiac death: the Task Force for the Management of Patients with Ventricular Arrhythmias and the Prevention of Sudden Cardiac Death of the European Society of Cardiology (ESC). Endorsed by: Association for European Paediatric and Congenital Cardiology (AEPC). Eur Heart J. 2015;36:2793-867. https://doi.org/10.1093/eurheartj/ehv316. 
33. Chen J, Todd DM, Proclemer A, Sciaraffia E, Estner HL, Broadhurst $\mathrm{P}$, et al. Management of patients with ventricular tachycardia in Europe: results of the European Heart Rhythm Association survey. Europace. 2015;17:1294-9. https://doi.org/ 10.1093/europace/euv255.

34. Tilz RR, Lenarczyk R, Scherr D, Haugaa KH, Iliodromitis K, Pürerfellner $\mathrm{H}$, et al. Management of ventricular tachycardia in the ablation era: results of the European Heart Rhythm Association Survey. Europace. 2018;20:209-13. https://doi.org/ 10.1093/europace/eux332.

35. Romero J, Stevenson WG, Fujii A, Kapur S, Baldinger SH, Mehta NK, et al. Impact of number of oral antiarrhythmic drug failures before referral on outcomes following catheter ablation of ventricular tachycardia. JACC Clin Electrophysiol. 2018;4:810-9. https:// doi.org/10.1016/j.jacep.2018.01.016.

36. Romero J, Di Biase L, Diaz JC, Quispe R, Du X, Briceno D, et al. Early versus late referral for catheter ablation of ventricular tachycardia in patients with structural heart disease: a systematic review and meta-analysis of clinical outcomes. JACC Clin Electrophysiol. 2018;4(3):374-82. https://doi.org/10.1016/j.jacep.2017.12.008 A meta-analysis on retrospective data on the benefits of an earlier referral.

37. Reddy VY, Reynolds MR, Neuzil P, Richardson AW, Taborsky M, Jongnarangsin $\mathrm{K}$, et al. Prophylactic catheter ablation for the prevention of defibrillator therapy. N Engl J Med. 2007 Dec 27;357: 2657-65. https://doi.org/10.1056/NEJMoa065457.

38. Kuck KH, Schaumann A, Eckardt L, Willems S, Ventura R, Delacrétaz E, et al. Catheter ablation of stable ventricular tachycardia before defibrillator implantation in patients with coronary heart disease (VTACH): a multicenter randomised controlled trial. Lancet. 2010 Jan 2;375:31-40. https://doi.org/10.1016/S01406736(09)61755-4.

39. Kuck KH, Tilz RR, Deneke T, Hoffmann BA, Ventura R, Hansen PS, et al. Impact of substrate modification by catheter ablation on implantable cardioverter-defibrillator interventions in patients with unstable ventricular arrhythmias and coronary artery disease: results from the multicenter randomized controlled SMS (Substrate Modification Study). Circ Arrhythm Electrophysiol. 2017;10: e004422. https://doi.org/10.1161/CIRCEP.116.004422.
40. Al-Khatib SM, Daubert JP, Anstrom KJ, Daoud EG, Gonzalez M, Saba $\mathrm{S}$, et al. Catheter ablation for ventricular tachycardia in patients with an implantable cardioverter defibrillator (CALYPSO) pilot trial. J Cardiovasc Electrophysiol. 2015;26:151-7. https:// doi.org/10.1111/jce.12567.

41. Martinez BK, Baker WL, Konopka A, Giannelli D, Coleman CI, Kluger J, et al. Systematic review and meta-analysis of catheter ablation of ventricular tachycardia in ischemic heart disease. Heart Rhythm. 2020 Jan;17:e206-19. https://doi.org/10.1016/j.hrthm. 2019.04.024.

42. Willems S, Tilz RR, Steven D, Kääb S, Wegscheider K, Gellér L, et al. Preventive or deferred ablation of ventricular tachycardia in patients with ischemic cardiomyopathy and implantable defibrillator (BERLIN VT): a multicenter randomized trial. Circulation. 2020;141:1057-67. https://doi.org/10.1161/ CIRCULATIONAHA.119.043400 The latest RCT on prophylactic catheter ablation.

43. Tzou WS, Tung R, Frankel DS, Di Biase L, Santangeli P, Vaseghi $\mathrm{M}$, et al. Outcomes after repeat ablation of ventricular tachycardia in structural heart disease: an analysis from the international VT ablation center collaborative group. Heart Rhythm. 2017 Jul;14:991-7. https://doi.org/10.1016/j.hrthm.2017.03.008.

44. Vaseghi M, Barwad P, Malavassi Corrales FJ, Tandri H, Mathuria $\mathrm{N}$, Shah R, et al. Cardiac sympathetic denervation for refractory ventricular arrhythmias. J Am Coll Cardiol. 2017;69:3070-80. https://doi.org/10.1016/j.jacc.2017.04.035.

45. Mazzone P, Peretto G, Radinovic A, Limite LR, Marzi A, Sala S, et al. The COVID-19 challenge to cardiac electrophysiologists: optimizing resources at a referral center. JICE. in press.

46. Sala S, Peretto G, Gramegna M, Palmisano A, Villatore A, Vignale $\mathrm{D}$, et al. Acute myocarditis presenting as a reverse Tako-Tsubo syndrome in a patient with SARS-CoV-2 respiratory infection. Eur Heart J. 2020. https://doi.org/10.1093/eurheartj/ehaa286.

47. Doyen D, Moceri P, Ducreux D, Dellamonica J. Myocarditis in a patient with COVID-19: a cause of raised troponin and ECG changes. Lancet. 2020. https://doi.org/10.1016/S0140-6736(20)30912-0.

Publisher's Note Springer Nature remains neutral with regard to jurisdictional claims in published maps and institutional affiliations. 